Louisiana State University

LSU Digital Commons

$1-1-2014$

\title{
Artemisia extracts activate PPARy, promote adipogenesis, and enhance insulin sensitivity in adipose tissue of obese mice
}

\author{
Allison J. Richard \\ Pennington Biomedical Research Center \\ Thomas P. Burris \\ Scripps Florida \\ David Sanchez-Infantes \\ Endocrinology Department \\ Yongjun Wang \\ Scripps Florida \\ David M. Ribnicky \\ Rutgers University-New Brunswick
}

See next page for additional authors

Follow this and additional works at: https://digitalcommons.Isu.edu/biosci_pubs

\section{Recommended Citation}

Richard, A., Burris, T., Sanchez-Infantes, D., Wang, Y., Ribnicky, D., \& Stephens, J. (2014). Artemisia extracts activate PPARY, promote adipogenesis, and enhance insulin sensitivity in adipose tissue of obese mice. Nutrition, 30 (7-8 SUPPL.) https://doi.org/10.1016/j.nut.2014.02.013 


\section{Authors}

Allison J. Richard, Thomas P. Burris, David Sanchez-Infantes, Yongjun Wang, David M. Ribnicky, and Jacqueline M. Stephens 


\title{
Artemisia extracts activate PPAR $\gamma$, promote adipogenesis, and enhance insulin sensitivity in adipose tissue of obese mice
}

\author{
Allison J. Richard ${ }^{1}$, Thomas P. Burris ${ }^{2}$, David Sanchez-Infantes ${ }^{3}$, Yongjun Wang ${ }^{2}$, David M. \\ Ribnicky ${ }^{4}$, and Jacqueline M. Stephens ${ }^{1,{ }^{*}}$ \\ ${ }^{1}$ Pennington Biomedical Research Center, Louisiana State University, Baton Rouge, LA 70808 \\ ${ }^{2}$ The Scripps Research Institute Jupiter, FL 33458 \\ ${ }^{3}$ Endocrinology Department, Saint Joan de Deu, Barcelona, Spain \\ ${ }^{4}$ Biotech Center, Rutgers University, New Brunswick, NJ 08901
}

\section{Abstract}

Objective-Studies have shown that the inability of adipose tissue to properly expand during the obese state or respond to insulin can lead to metabolic dysfunction. Artemisia is a diverse group of plants that has a history of medicinal use. This study examines the ability of ethanolic extracts of Artemisia scoparia (SCO) and Artemisia santolinifolia (SAN) to modulate adipocyte development in cultured adipocytes and white adipose tissue (WAT) function in vivo using a mouse model of diet-induced obesity.

\begin{abstract}
Research Design \& Procedures-Adipogenesis was assessed using Oil Red O staining and immunoblotting. A nuclear receptor specificity assay was used to examine the specificity of SCOand SAN-induced PPAR $\gamma$ activation. C57BL/6J mice, fed a high-fat diet, were gavaged with saline, SCO, or SAN for 2 weeks. Whole-body insulin sensitivity was examined using insulin tolerance tests. WAT depots were assessed via immunoblotting for markers of insulin action and adipokine production.
\end{abstract}

Results-We established that SCO and SAN were highly specific activators of PPAR $\gamma$ and did not activate other nuclear receptors. After a one-week daily gavage, SCO- and SAN-treated mice had lower insulin-induced glucose disposal rates than control mice. At the end of the 2-week treatment period, SCO- and SAN-treated mice had enhanced insulin-responsive Akt serine-473 phosphorylation and significantly decreased MCP-1 levels in visceral WAT relative to control

\footnotetext{
(C) 2014 Elsevier Inc. All rights reserved.

* To whom correspondence should be addressed, Louisiana State University, Pennington Biomedical Research Center, Baton Rouge, LA 70803, Phone (225)-763-2648, FAX (225)-578-2597, jsteph1@1su.edu.

Publisher's Disclaimer: This is a PDF file of an unedited manuscript that has been accepted for publication. As a service to our customers we are providing this early version of the manuscript. The manuscript will undergo copyediting, typesetting, and review of the resulting proof before it is published in its final citable form. Please note that during the production process errors may be discovered which could affect the content, and all legal disclaimers that apply to the journal pertain.

Disclosure Statement:

The authors have no potential conflicts of interest to disclose.

Author Contributions:

AJR, TPB, and JMS designed the experiments; AJR, YW, and DS-I processed and analyzed data; DMR provided the Artemisia extracts; AJR and JMS interpreted the data and wrote the manuscript. All authors contributed to preparation of the final manuscript.
} 
mice; these differences were depot specific. Moreover, plasma adiponectin levels were increased following SCO treatment.

Conclusion-Overall, these studies demonstrate that extracts from two Artemisia species can have metabolically favorable effects on adipocytes and WAT.

\section{Keywords}

fat cells; insulin action; botanicals; Diet Induced Obesity; 3T3-L1 adipocytes

\section{Introduction}

Adipocytes are dynamic insulin-sensitive cells that have endocrine properties and contribute to whole body energy homeostasis. Obesity is the primary disease of fat cells and significantly contributes to the development of type 2 diabetes mellitus (T2DM), cardiovascular disease, and certain cancers. Many researchers have investigated antiadipogenic agents, including some botanicals, as potential therapeutics for decreasing or preventing obesity. However, the prevailing current hypothesis is that disruption of adipocyte differentiation limits adipose tissue expansion and leads to insulin resistance and the development of T2DM [1-4].

Botanical extracts represent an alternative approach for the treatment or prevention of a disease. Plant extracts are used by many cultures and have resulted in the development of many drugs, including metformin. Metformin is a widely used T2DM drug that is derived from French lilac. In a blinded screening study to investigate the effects of botanicals on adipocyte differentiation, we identified plant extracts with substantial effects on adipogenesis. Two of these extracts were Artemisia species, Artemisia scoparia (SCO) and Artemisia santolinifolia (SAN). Many Artemisia species have been used in traditional medicine in East Asia, and are reported to demonstrate anti-hyperglycemic [5, 6], antiobesity [7, 8], and anti-diabetic activities [5, 9]. Our studies revealed that both SCO and SAN could promote adipocyte development in differentiating murine adipocytes. In addition, these extracts specifically activate PPAR $\gamma$, but did not modulate the activity of other nuclear receptors. We also observed that SCO and SAN could increase adiponectin (ADPN) secretion in vitro and in vivo. In a 2-week gavage study, SCO and SAN enhanced insulin action in epididymal adipose tissue. These studies demonstrate that SCO and SAN have a positive impact on adipocyte-related diseases by promoting adipocyte development and increasing adiponectin levels and insulin action in adipose tissue.

\section{Materials and Methods}

\section{Chemicals and reagents}

Dulbecco's modified Eagle's medium (DMEM) was purchased from Sigma-Aldrich (St. Louis, MO). Bovine and fetal bovine sera were purchased from HyClone (Thermo Scientific, Logan, UT). For immunoblotting, STAT5A, MAPK/ERK, and PPAR $\gamma$ antibodies were purchased from Santa Cruz Biotechnology (Santa Cruz, CA). The anti-adiponectin antibody was obtained from Thermo Scientific (Rockford, IL). Anti-phospho-Akt$\mathrm{Ser}^{473}(\operatorname{AktpS}(473))$, total Akt, and monocyte chemotactic protein-1 (MCP-1) antibodies 
were purchased from Cell Signaling Technology, Inc. (Danvers, MA). With the exception of the mouse monoclonal anti-PPAR $\gamma \operatorname{IgG}$, all other antibodies were rabbit polyclonal IgGs, and peroxidase-conjugated secondary antibodies to both species were purchased from Jackson ImmunoResearch (West Grove, PA). The BCA and enhanced chemiluminescence kits were from Thermo Scientific.

\section{Preparation, source and characterization of the extracts}

Artemisia scoparia Waldst. \& Kit and Artemisia santolinifolia Turcz. ex Besser ethanolic extracts were prepared at Rutgers University. Briefly, the herb was greenhouse grown from seed and periodically harvested at the flowering stage, freeze dried and stored at $-20^{\circ} \mathrm{C}$. The dried herb was extracted in $80 \%$ ethanol $(1: 20 \mathrm{w} / \mathrm{v})$, at $50^{\circ} \mathrm{C}$ with sonication for 1 hour followed by shaking at room temperature for $24 \mathrm{~h}$. The solid material was removed by centrifugation at $3000 \mathrm{~g}$ and the solvent was subsequently removed by evaporation. For in vitro and cell culture experiments, the dried extracts were solubilized in 100\% DMSO at a concentration that was 1000-fold higher than experimental concentrations and then diluted into the media. For animal studies, the extracts were solubilized in $20 \%$ Labrasol $^{\circledR}$ (Gattefosse, Lyon, France) and administered via gavage.

\section{Nuclear receptor specificity assay}

SCO and SAN were assessed for their ability to modulate the activities of all 48 human nuclear receptors using a previously described Gal4 co-transfection assay system [10-12]. Both extracts were tested at $50 \mu \mathrm{g} / \mathrm{ml}$ and positive controls were included for characterized ligands. DMSO was used as the vehicle control.

\section{Cell culture}

Murine 3T3-L1 preadipocytes were plated and grown to 2 days post-confluence in DMEM containing $10 \%$ bovine serum. Medium was changed every 48-72 hours. Cells were induced to differentiate using a standard MDI induction protocol [13]. Mature adipocytes were maintained in DMEM supplemented with 10\% FBS until utilized for experimentation. For adipogenesis assays, the botanical extracts were added at the time of MDI induction and at each media change until the cells were stained with Oil Red O (ORO) or harvested for western blot analysis.

\section{Oil Red O staining}

An ORO stock was prepared as previously described [14]. Cell monolayers were aspirated, rinsed with PBS, fixed in 10\% formaldehyde/PBS, and rinsed under tap water. The remaining water was aspirated, and the cells were incubated for $1 \mathrm{~h}$ in the working ORO solution ( $0.3 \%$ in isopropanol). Following incubation, stain aspiration, and rinsing, cells were examined by microscopy and scanned to produce the figures in this manuscript.

\section{Animals and gavage}

Thirty-six 16-week old male C57BL/6J-diet-induced obese (DIO) mice were purchased from Jackson Laboratories (Bar Harbor, ME) and maintained on a purified high-fat diet (HFD) containing $20 \mathrm{kcal} \%$ protein, $20 \mathrm{kcal} \%$ carbohydrate, and $60 \mathrm{kcal} \%$ fat (D12492; 
Research Diets, Inc., New Brunswick, NJ) for the entire study. After one week in quarantine, mice were housed four per cage in a temperature- and humidity-controlled room with a 12-hour light/dark cycle. Mice were handled daily for 2 weeks and non-fasting body weights were recorded daily. Non-fasting NMR (Bruckner Minispec) was recorded on day 14. Animals were gavaged (22 gauge plastic gavage needle, Instech Laboratories, Inc. Plymouth Meeting, PA) with $20 \%$ Labrasol $^{\circledR}$ daily for 7 days. On day 21 , another nonfasting NMR measurement was recorded and submandibular blood samples were collected. Animals were randomized by non-fasting body weight (day 21) to one of 3 groups: $20 \%$ Labrasol ${ }^{\circledR}(\mathrm{CTL} ; \mathrm{n}=12), \mathrm{SCO}(\mathrm{n}=12)$, and SAN $(\mathrm{n}=12)$ and gavaged daily for an additional 2 weeks. Botanical extracts were prepared in $20 \%$ Labrasol $^{\circledR}$ to increase bioavailability. All animal studies were performed with approval from the Pennington Biomedical Research Center Institutional Animal Care and Use Committee.

\section{Animal Study Procedures}

Non-fasted body weight was measured daily during the 2-week gavage study. Body composition (NMR), fasting body weight, fasting plasma glucose, insulin, and adiponectin levels were measured at the beginning (baseline measurements) and end of the 2-week study period. One week following the initiation of experimental gavaging, and 4 hours after mice received their last gavage, intraperitoneal insulin tolerance tests (IPITTs) were performed on all animals. Briefly, a baseline blood glucose measurement was obtained via tail nick ( 0 minutes), and animals were then injected with $1 \mathrm{U} / \mathrm{kg}$ insulin (Humulin R, Eli Lilly, Indianapolis, IN). Blood glucose measurements were obtained via tail nick at 10, 20, 40, and 60 minutes post-injection. All blood glucose measurements were performed using a Bayer Breeze 2 glucometer. Following 2 weeks of experimental gavage, mice were fasted overnight and a fasted body weight, submandibular blood collection, and NMR measurement were obtained for each mouse. Immediately following these procedures, mice were gavaged with the appropriate extract or vehicle. Four hours after the gavage, mice were injected with either $1 \mathrm{U} / \mathrm{kg}$ insulin or saline and euthanized via cervical dislocation 10-15 minutes after injections. Animals were then decapitated and trunk blood collected. Retroperitoneal white adipose tissue (rWAT), inguinal adipose tissue (iWAT), and epididymal adipose tissue (eWAT) depots were collected and immediately frozen in liquid nitrogen.

\section{Whole cell extract and tissue preparation}

Adipocyte whole cell extracts and WAT lysates were prepared by either harvesting adipocyte monolayers or homogenizing WAT in a non-denaturing extraction buffer containing $150 \mathrm{mM} \mathrm{NaCl}, 10 \mathrm{mM}$ Tris, pH 7.4, 1 mM EGTA, $1 \mathrm{mM}$ EDTA, $1 \%$ Triton $\mathrm{X}-100,0.5 \%$ Igepal CA-630, $1 \mathrm{mM}$ phenylmethylsulfonyl fluoride, $1 \mu \mathrm{M}$ pepstatin, 50 trypsin inhibitory milliunits of aprotinin, $10 \mu \mathrm{M}$ leupeptin, $1 \mathrm{mM}$ 1, 10-phenanthroline, and $0.2 \mathrm{mM}$ sodium vanadate. Protein content was determined by BCA protein assay (Bio-Rad laboratories, Inc., Hercules, CA).

\section{Gel electrophoresis and immunoblotting}

Samples were separated on 7.5, 10, or 15\% SDS-polyacrylamide gels (PA) and transferred to nitrocellulose membranes. Results were visualized with horseradish peroxidase- 
conjugated secondary antibodies and enhanced chemiluminescence. For native gel electrophoresis, 5\% PA gels were prepared without SDS. Additionally, SDS and reducing agents were eliminated from the running and sample loading buffers.

\section{Statistics}

Data were graphed as mean \pm SEM. The differences between treatment means were tested using an ANOVA. Pairwise t-tests were performed to determine which means were different when the ANOVA was statistically significant. A mixed model was used to model blood glucose. Time, as a categorical variable, treatment, and time by treatment interaction were included in the model. T-tests were performed at each time point on the least square means from the model to determine if there were any statistically significant differences between the treatments. All tests used $p<0.05$ as the level of statistical significance.

\section{Results}

\section{Artemisia extracts act as PPAR $\gamma$ agonists and promote adipogenesis of 3T3-L1 cells}

We screened over 400 botanical extracts for their ability to activate PPAR $\gamma$ and modulate adipogenesis in 3T3-L1 cells. Our results identified SCO and SAN as modulators of adipocyte development. As shown in Figure 1A, SCO and SAN increased ORO staining of 3T3-L1 cells during adipogenesis relative to the vehicle control. Artemisia rutifolia and GOP (grains of paradise) were not capable of promoting lipid accumulation relative to the vehicle control. We also examined the ability of these botanical extracts to modulate the expression of STAT5A and other adipocyte marker proteins, such as PPAR $\gamma$ and ADPN, which are induced during fat cell differentiation. SCO and SAN substantially increased the expression levels of the adipogenic marker proteins in 3T3-L1 adipocytes (Figure 1B). Consistent with ORO results, addition of A. rutifolia did not increase PPAR $\gamma$, adiponectin, or STAT5A levels. Thus, SCO and SAN were selected for further investigation.

PPAR $\gamma$ belongs to the large nuclear receptor (NR) superfamily. We examined the ability of SCO and SAN to modulate the activity of all forty-eight known human NRs by utilizing a NR specificity assay. As previously demonstrated [11], activation and repression of NRs were denoted as increases or decreases (greater than 3 standard deviations), respectively, in fold change versus DMSO vehicle. SCO repressed and SAN stimulated the transactivating activity of the many of the NR ligand binding domains (LBDs), but these marginal modulations were not significant. However, SCO and SAN were capable of significantly stimulating PPAR $\gamma$. As shown in Figure 2, both SCO and SAN significantly activated the LBD of PPAR $\gamma$ greater than 2 fold, and thus were extremely selective PPAR $\gamma$ agonists.

\section{Physical and metabolic parameters of HFD-fed C57BL/6 mice treated with SCO and SAN}

To investigate the ability of SCO and SAN to modulate adipocyte function in vivo, we used the DIO C57BL/6J mouse model. SCO and SAN $(500 \mathrm{mg} / \mathrm{kg}$ ) were administered to male mice daily via gavage for a period of two weeks. No significant effects on body weight, physical appearance and activity were observed as a result of pre-treatment with Labrasol ${ }^{\circledR}$ for one week. There were no significant differences in fasting body weight, fat mass (FM), fat free mass (FFM), or the ratio of fat mass to lean mass (FM/LM) between the CTL and 
SCO or SAN-treated mice at the end of the two-week treatment (data not shown). However, as shown in Figure 3, SCO-treated mice displayed increased insulin-induced glucose disposal relative to control mice during an IPITT. Differences in the blood glucose levels of SCO and CTL mice were statistically significant at the 20 and 60 min time points. Mice treated with SAN also displayed slightly improved insulin-sensitive glucose disposal, especially at time points between 20 and 60 minutes.

\section{SCO and SAN increase insulin sensitivity in WAT in a depot specific manner}

Following two weeks of botanical treatment, the mice were fasted overnight, and then injected with saline or insulin 10 minutes prior to sacrifice. eWAT, rWAT, and iWAT were collected and analyzed for Akt S473 phosphorylation. As shown in Figure 4, saline-injected animals exhibited little to no AktpS(473) in any of the WAT depots. In response to insulin, SCO- and SAN-treated mice displayed improved insulin sensitivity in eWAT when compared to controls, as indicated by higher levels of AktpS(473). In rWAT and iWAT, AktpS(473) levels were similar among all groups. Total Akt protein levels were not altered by botanical treatment in any WAT depot.

\section{SCO and SAN promote a beneficial adipokine profile in DIO mice}

We also examined the effects of SCO and SAN on plasma ADPN levels. Plasma levels of high molecular weight (HMW) and total ADPN were similar in SAN-treated and control animals. SCO-treated mice exhibited elevated circulating levels of both total and HMW ADPN (Figure 5) relative to controls, but this difference was not statistically significant. We also examined MCP-1 protein levels in the WAT lysates. As shown in Figure 6, we observed significant decreases in MCP-1 protein expression in the rWAT of SCO- and SAN-treated mice relative to control animals. This trend was also observed in eWAT, but not in iWAT (data not shown).

\section{Discussion}

Alterations in adipocytes and/or adipose tissue can have profound effects on metabolic disease states. Improvements in adipocyte differentiation and adipocyte function represent viable interventions for metabolic disease states including T2DM. For these reasons, we examined the effects of a variety of botanical extracts on adipocyte development and function. In a blinded screening study, we observed that ethanolic extracts from two related Artemisia species, Artemisia scoparia and Artemisia santolinifolia can promote adipocyte development in vitro. Moreover, extracts from these Artemisia species specifically activated PPAR $\gamma$, a transcription factor known to promote adipocyte development in vitro and in vivo $[15,16]$. Gavage studies in DIO mice were consistent with our in vitro observations and indicated that SCO and SAN treated animals had reduced glucose levels following an insulin tolerance test. We also observed increase insulin sensitivity as measured by Akt phosphorylation in epididymal fat, but not other white fat depots. Adiponectin is a hormone associated with cardioprotection and insulin sensitivity in mice in man $[17,18]$ and we observed increases globular and high molecular weight adiponectin in mice treated with SCO and SAN. These observations are consistent with the results of the insulin tolerance test and examination of insulin action in adipose tissue. We predict the ability of SCO and 
SAN to improve insulin action may be mediated by its ability to limit inflammation in adipose tissue as our studies revealed that both Artemisia species reduced the expression of MCP-1 in adipose tissue. Although other Artemisia species have been shown to have antidiabetic [5-7, 19] and insulin-sensitizing [20-22] activities, this is the first demonstration that Artemisia SCO and SAN species can modulate adipocyte development and function. There is also evidence to suggest that Artemisia scoparia can attenuate lipid accumulation in liver and enhance hepatic insulin sensitivity [23]. Overall, our studies demonstrate that two related Artemisia species have a positive impact on adipocyte related diseases by enhancing differentiation of preadipocytes and increasing insulin sensitivity in adipose tissue in vivo.

\section{Acknowledgments}

We would like to thank Anik Boudreau for technical assistance, Dr. Carrie Elks for assistance with manuscript preparation, and Drs. William Johnson and Dr. Robbie Beyl for assistance with statistical analyses. Also, we gratefully acknowledge Dr. Randy Mynatt and Tamra Mendoza in the Integrative Biology Core of our Botanical Research Center for help with the gavage studies.

Funding:

NCAAM and ODS funded NIH grant-2P50AT002776-06. AJR was supported by an NIH T-32 postdoctoral training fellowship in botanicals granted to Pennington Biomedical Research Center (T32 AT004094).

\section{Abbreviations}

T2DM

SCO

SAN

$\operatorname{PPAR} \gamma$

ADPN

DMEM

STAT5A

MAPK

MCP-1

BCA

DMSO

FBS

MDI

ORO

DIO

HFD

NMR

IPITT
Type 2 Diabetes Mellitus

Artemisia scoparia extracts

Artemisia santolinifolia extracts

peroxisome proliferator-activated receptor gamma

adiponectin

Dulbecco's Modified Eagle's Medium

signaltransducer and activator of transcription 5

mitogen-activated protein kinase

monocyte chemoattractant protein-1

bicinchoninic acid

dimethyl sulfoxide

fetal bovine serum

1-methyl-3-isobutylxanthine, dexamethasone, and insulin

Oil Red O

diet induced obesity

high fat diet

nuclear magnetic resonance

intraperitoneal insulin tolerance test 
i, e, and r WAT inguinal, epididymal, and retroperitoneal white adipose tissue

SDS

PA

ANOVA sodium dodecyl sulfate

polyacrylamide

ANalysis Of VAriance between groups

\section{Reference List}

1. Danforth E Jr. Failure of adipocyte differentiation causes type II diabetes mellitus? Nat.Genet. 2000; 26:13. [PubMed: 10973236]

2. Kim JY, van de Wall E, Laplante M, et al. Obesity-associated improvements in metabolic profile through expansion of adipose tissue. J.Clin.Invest. 2007; 117:2621-2637. [PubMed: 17717599]

3. Richard AJ, Amini-Vaughan Z, Ribnicky DM, et al. Naringenin inhibits adipogenesis and reduces insulin sensitivity and adiponectin expression in adipocytes. Evid.Based.Complement Alternat.Med. 2013; 2013:549750. [PubMed: 23983791]

4. Khan T, Muise ES, Iyengar P, et al. Metabolic dysregulation and adipose tissue fibrosis: role of collagen VI. Mol.Cell Biol. 2009; 29:1575-1591. [PubMed: 19114551]

5. Xing XH, Zhang ZM, Hu XZ, et al. Antidiabetic effects of Artemisia sphaerocephala Kraschgum, a novel food additive in China, on streptozotocin-induced type 2 diabetic rats. J Ethnopharmacol. 2009; 125:410-416. [PubMed: 19635546]

6. Ribnicky DM, Poulev A, Watford M, et al. Antihyperglycemic activity of Tarralin, an ethanolic extract of Artemisia dracunculus L. Phytomedicine. 2006; 13:550-557. [PubMed: 16920509]

7. Yamamoto N, Kanemoto Y, Ueda M, et al. Anti-obesity and anti-diabetic effects of ethanol extract of Artemisia princeps in C57BL/6 mice fed a high-fat diet. Food Funct. 2011; 2:45-52. [PubMed: 21773585]

8. Lim DW, Kim YT, Jang YJ, et al. Anti-obesity effect of Artemisia capillaris extracts in high-fat diet-induced obese rats. Molecules. 2013; 18:9241-9252. [PubMed: 23917113]

9. Hamza N, Berke B, Cheze C, et al. Prevention of type 2 diabetes induced by high fat diet in the C57BL/6J mouse by two medicinal plants used in traditional treatment of diabetes in the east of Algeria. J Ethnopharmacol. 2010; 128:513-518. [PubMed: 20064599]

10. Busby SA, Kumar N, Kuruvilla DS, et al. Identification of a novel non-retinoid pan inverse agonist of the retinoic acid receptors. ACS Chem.Biol. 2011; 6:618-627. [PubMed: 21381756]

11. Kumar N, Solt LA, Conkright JJ, et al. The benzenesulfoamide T0901317 [N-(2,2,2trifluoroethyl)-N-[4-[2,2,2-trifluoro-1-hydroxy-1-(trifluoromethyl)ethy 1]phenyl]benzenesulfonamide] is a novel retinoic acid receptor-related orphan receptor-alpha/gamma inverse agonist. Mol.Pharmacol. 2010; 77:228-236. [PubMed: 19887649]

12. Solt LA, Wang Y, Banerjee S, et al. Regulation of circadian behaviour and metabolism by synthetic REV-ERB agonists. Nature. 2012; 485:62-68. [PubMed: 22460951]

13. Richard AJ, Amini ZJ, Ribnicky DM, et al. St. John's Wort inhibits insulin signaling in murine and human adipocytes. Biochim Biophys Acta. 2012; 1822:557-563. [PubMed: 22198320]

14. Kuri-Harcuch W, Green H. Adipose conversion of $3 \mathrm{~T} 3$ cells depends on a serum factor. Proc.Natl Acad.Sci U.S.A. 1978; 75:6107-6109. [PubMed: 282628]

15. Spiegelman BM, Hu E, Kim JB, et al. PPAR gamma and the control of adipogenesis. Biochimie. 1997; 79:111-112. [PubMed: 9209705]

16. Farmer SR. Transcriptional control of adipocyte formation. Cell Metab. 2006; 4:263-273. [PubMed: 17011499]

17. Hopkins TA, Ouchi N, Shibata R, et al. Adiponectin actions in the cardiovascular system. Cardiovasc.Res. 2007; 74:11-18. [PubMed: 17140553]

18. Ye R, Scherer PE. Adiponectin, driver or passenger on the road to insulin sensitivity? Mol Metab. 2013; 2:133-141. [PubMed: 24049728] 
19. Hamza N, Berke B, Cheze C, et al. Treatment of high fat diet induced type 2 diabetes in C57BL/6J mice by two medicinal plants used in traditional treatment of diabetes in the east of Algeria. $\mathrm{J}$ Ethnopharmacol. 2011; 133:931-933. [PubMed: 21094236]

20. Obanda DN, Hernandez A, Ribnicky D, et al. Bioactives of Artemisia dracunculus L. mitigate the role of ceramides in attenuating insulin signaling in rat skeletal muscle cells. Diabetes. 2012; 61:597-605. [PubMed: 22315320]

21. Wang ZQ, Ribnicky D, Zhang XH, et al. Bioactives of Artemisia dracunculus L enhance cellular insulin signaling in primary human skeletal muscle culture. Metabolism. 2008; 57:S58-S64. [PubMed: 18555856]

22. Wang ZQ, Ribnicky D, Zhang XH, et al. An extract of Artemisia dracunculus L. enhances insulin receptor signaling and modulates gene expression in skeletal muscle in KK-A(y) mice. J Nutr Biochem. 2011; 22:71-78. [PubMed: 20447816]

23. Wang ZQ, Zhang XH, Yu Y, et al. Artemisia scoparia extract attenuates non-alcoholic fatty liver disease in diet-induced obesity mice by enhancing hepatic insulin and AMPK signaling independently of FGF21 pathway. Metabolism. 2013; 62:1239-1249. [PubMed: 23702383] 
A

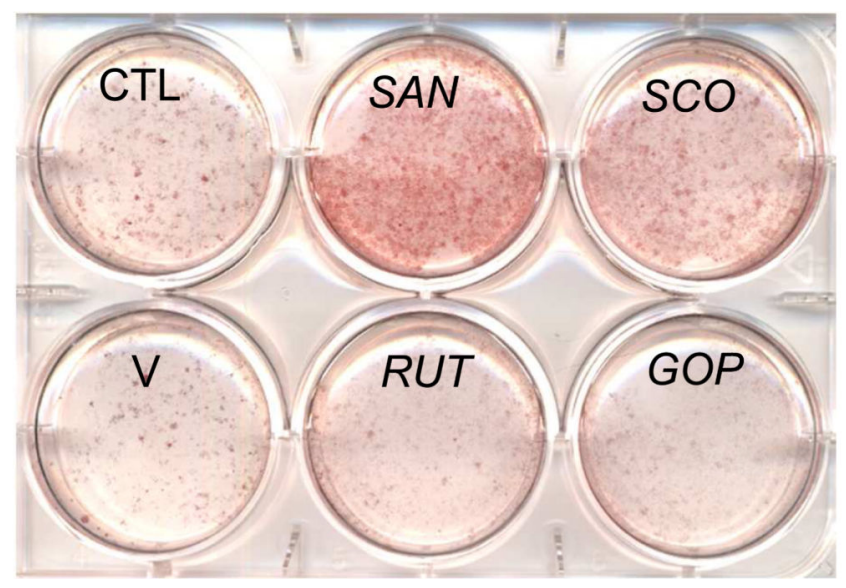

B

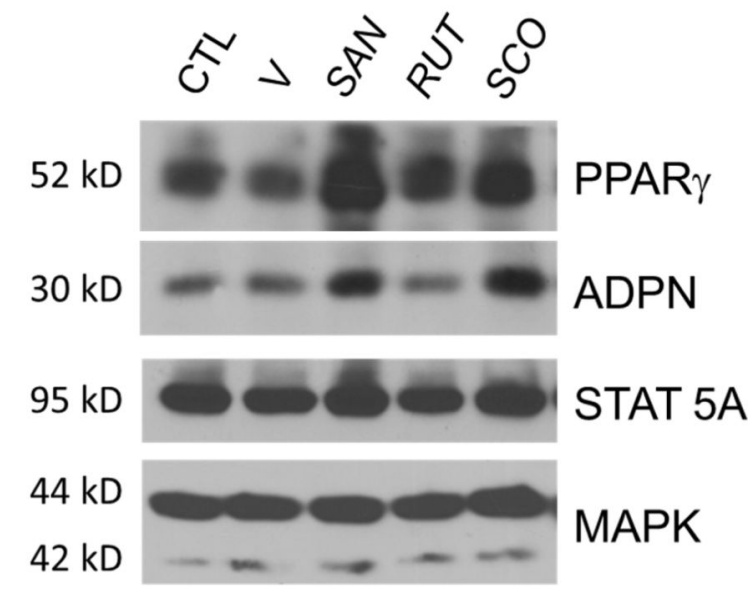

Figure 1. SCO and SAN promote adipogenesis of 3T3-L1 cells

Differentiation of murine 3T3-L1 preadipocytes was induced using the standard induction cocktail containing $50 \mu \mathrm{g} / \mathrm{ml}$ of the indicated extracts (Artemisia rutifolia; RUT and Grains of Paradise; GOP), untreated control (CTL), or DMSO vehicle (V). A) Cell monolayers were subjected to ORO staining one week after induction. B) Western blot analysis was performed on whole cell extracts $(100 \mu \mathrm{g}$ protein) prepared from cells that were harvested one week after induction. The results represent three independently performed experiments. 

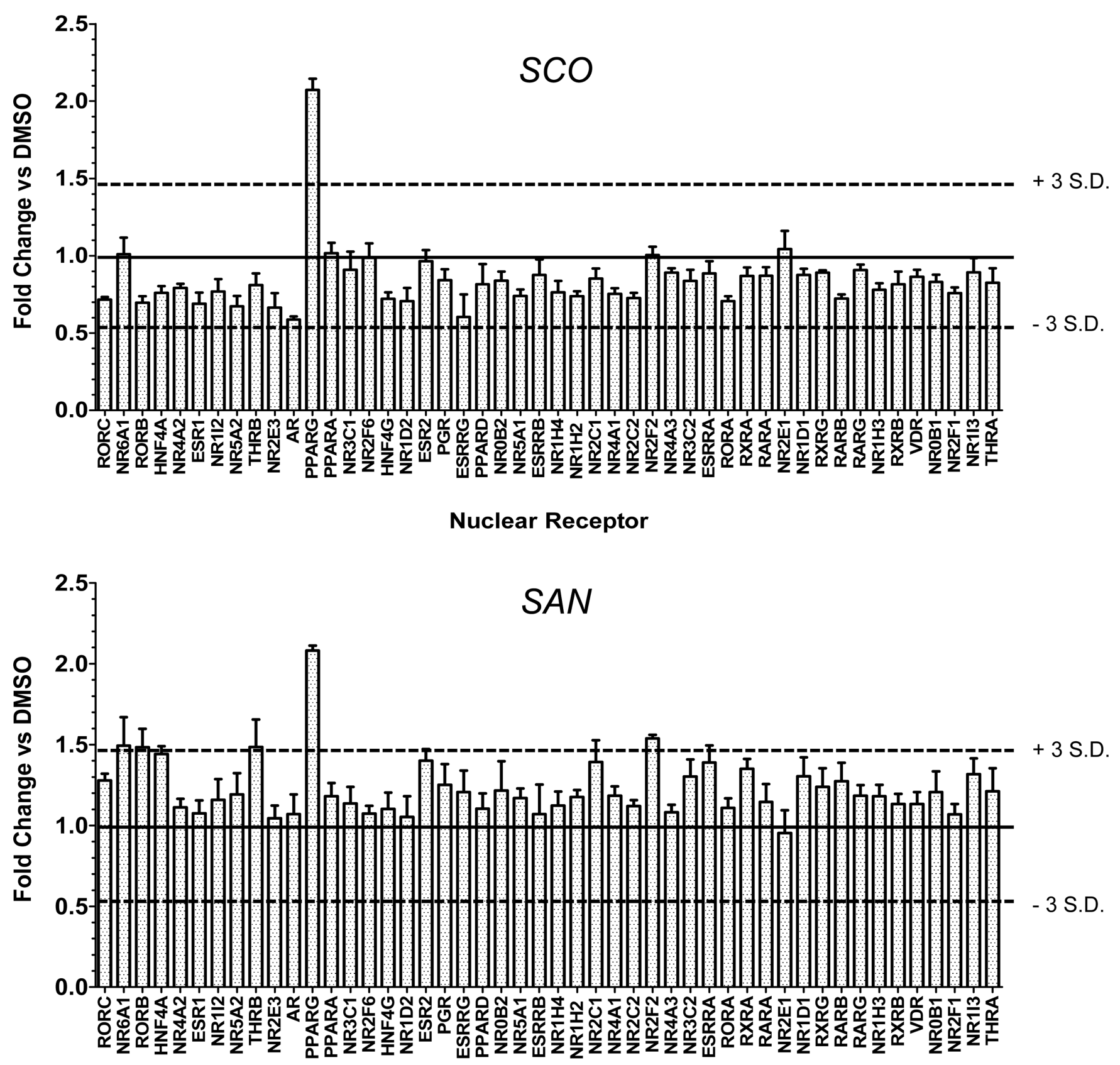

Nuclear Receptor

Figure 2. SCO and SAN specifically activate PPAR $\gamma$

The ability of SCO and SAN to modulate the transactivation all 48 human NRs was assessed using a NR specificity assay. HEK293 cells were co-transfected with Gal4 DNA binding domain - NR fusions and luciferase reporter constructs. Extracts were tested at $50 \mu \mathrm{g} / \mathrm{ml}$, and DMSO was used as a vehicle control. The luciferase activity of each construct was measured and normalized to the mock (vector alone), and then the fold change in signal compared with DMSO was calculated $(n=4)$. Data are means $+/-$ SEM $(n=4)$. Horizontal 
dashed lines represent + /- 3 S.D. of the luciferase activity measured following DMSO treatment. 


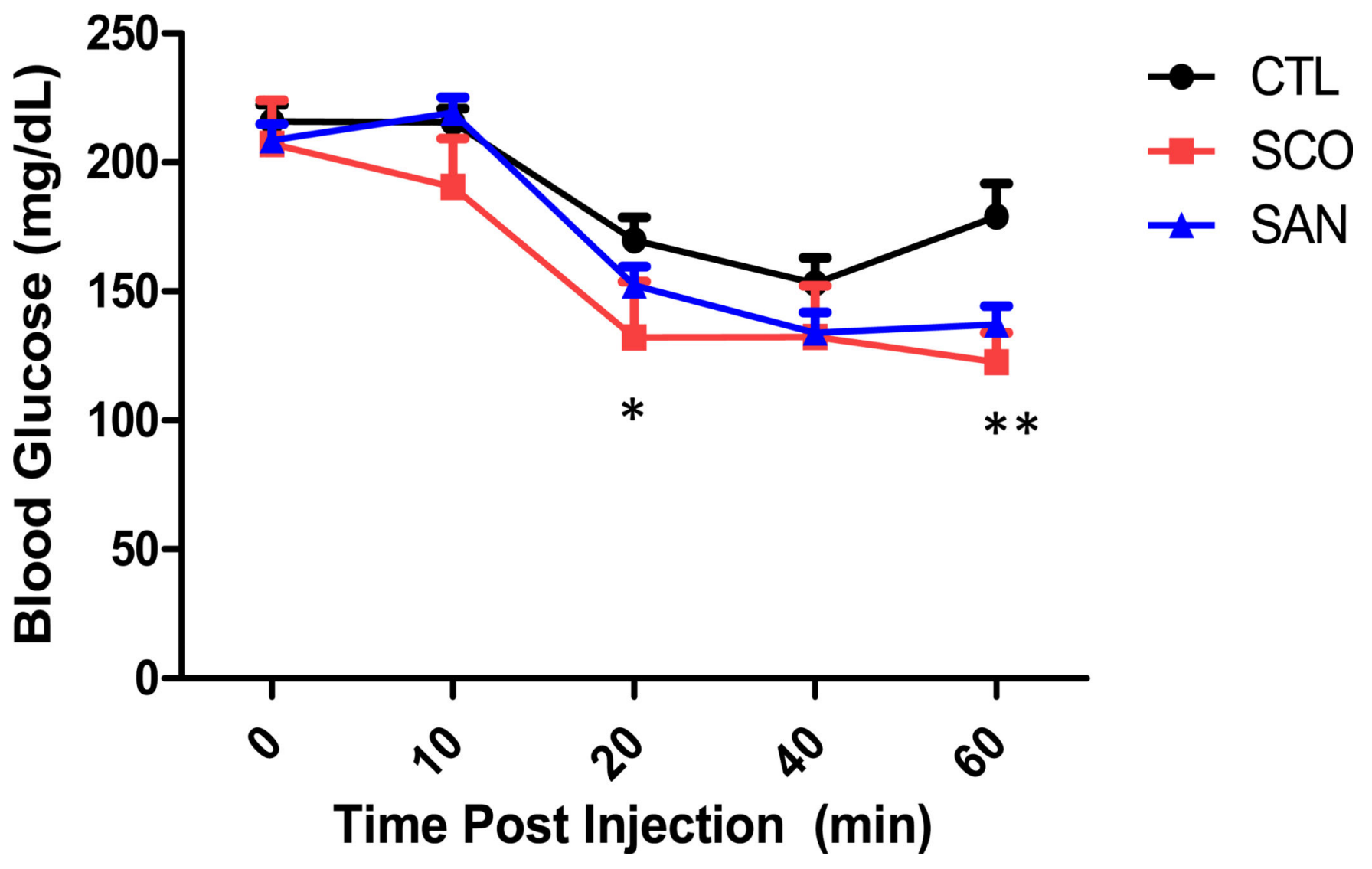

Figure 3. SCO and SAN improve glucose disposal in response to an intraperitoneal insulin injection

Mice were gavaged with $20 \%$ Labrasol $^{\circledR}$ (CTL) in the presence or absence of SCO or SAN daily for one week. Blood glucose levels were monitored at the indicated time points following a single insulin injection of insulin. Data are presented as mean $+/-$ SEM $(n=12)$. $* \mathrm{p} \leq 0.03, \mathrm{SCO}$ versus CTL and $* * \mathrm{p} \leq 0.01$, SCO or SAN versus CTL. 

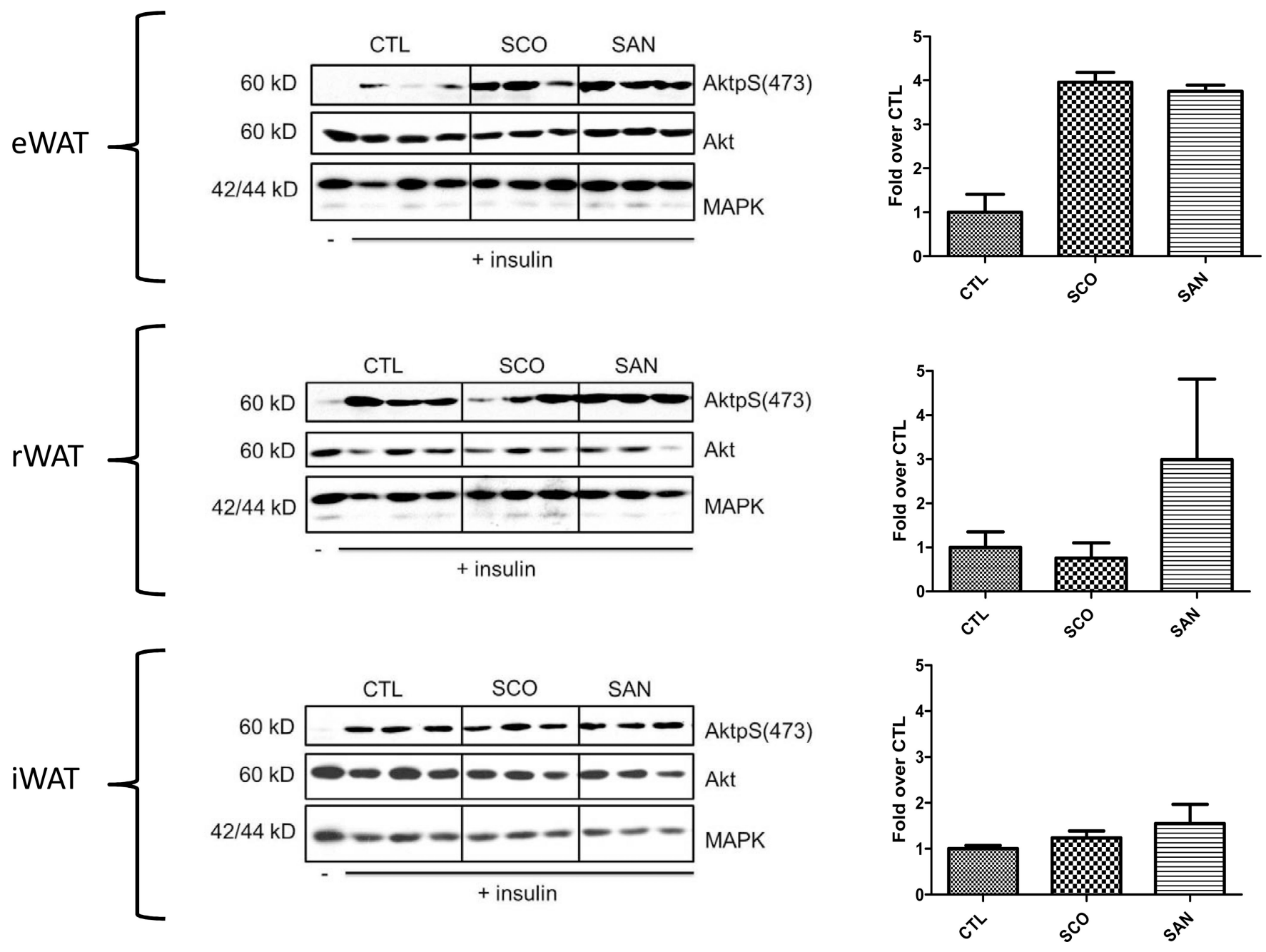

Figure 4. SCO and SAN increase insulin-induced Akt phosphorylation in eWAT

Tissue extracts of eWAT, iWAT, and rWAT from CTL-, SCO- and SAN-treated mice were subjected to Western blot analysis (50-150 $\mu$ g protein). There were 6 mice per condition and three animals per condition are shown. For each depot, the results for each antibody are from the same exposure of the same blot. Densitometric analyses are shown to the right of each blot; AktpS(473) band intensities were normalized by the respective total Akt band intensities prior to comparison against CTL. Data are means $+/-$ SEM $(n=3)$. 

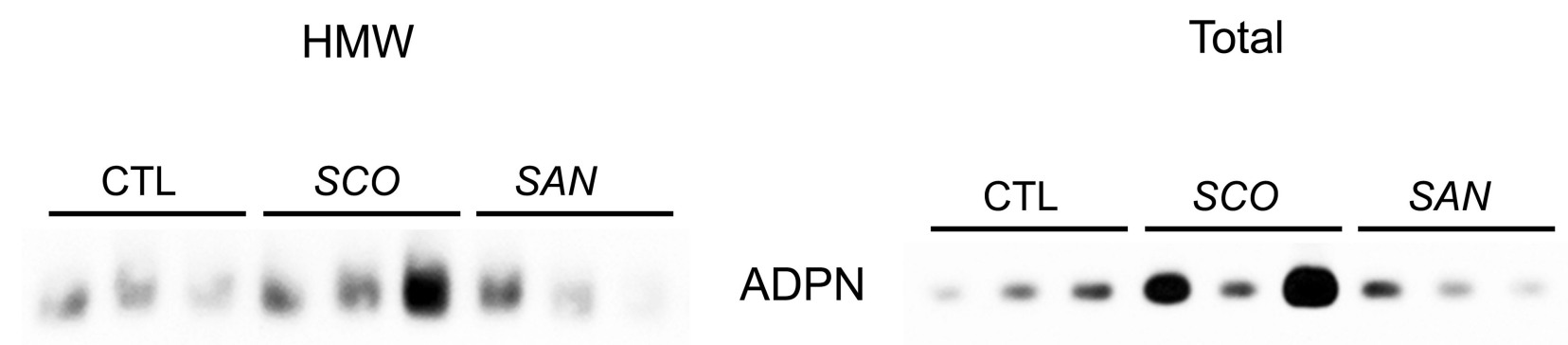

$225 \mathrm{kD}$
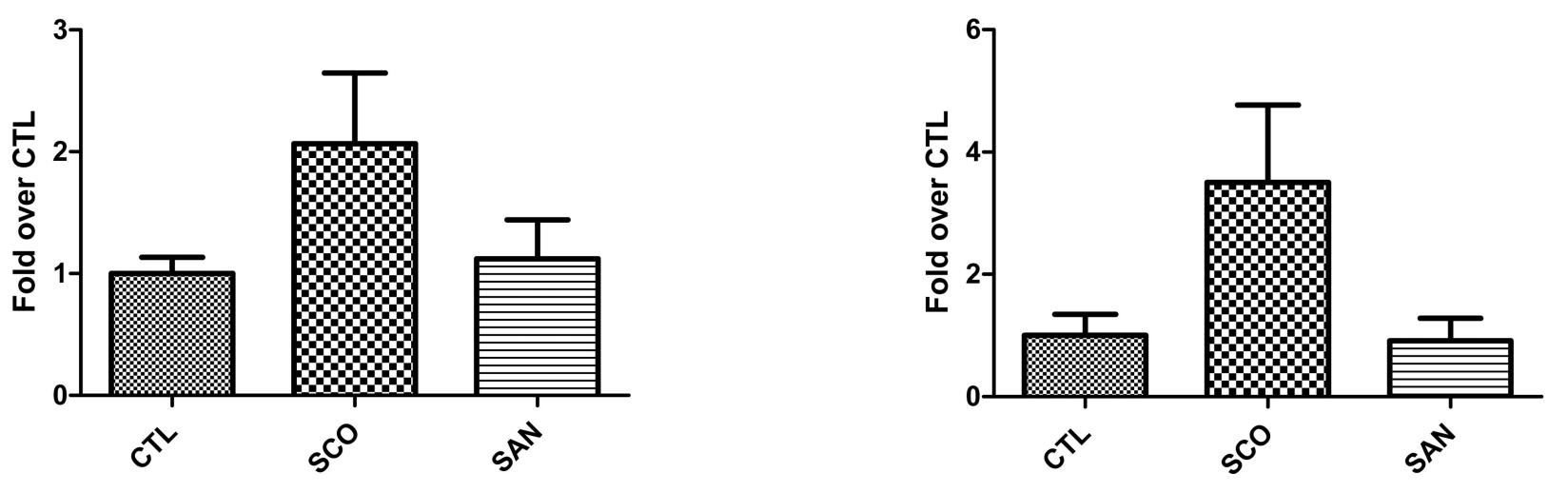

Figure 5. SCO increases the plasma levels of HMW and total ADPN in DIO mice

Serum HMW and total ADPN, were separated by native PA gel electrophoresis ( $50 \mu \mathrm{g}$ protein) and SDS-PAGE, respectively, for HMW and total protein content, and visualized by Western blot analysis. Densitometric analysis is shown below each blot; Data are means +/$\operatorname{SEM}(n=3)$. 

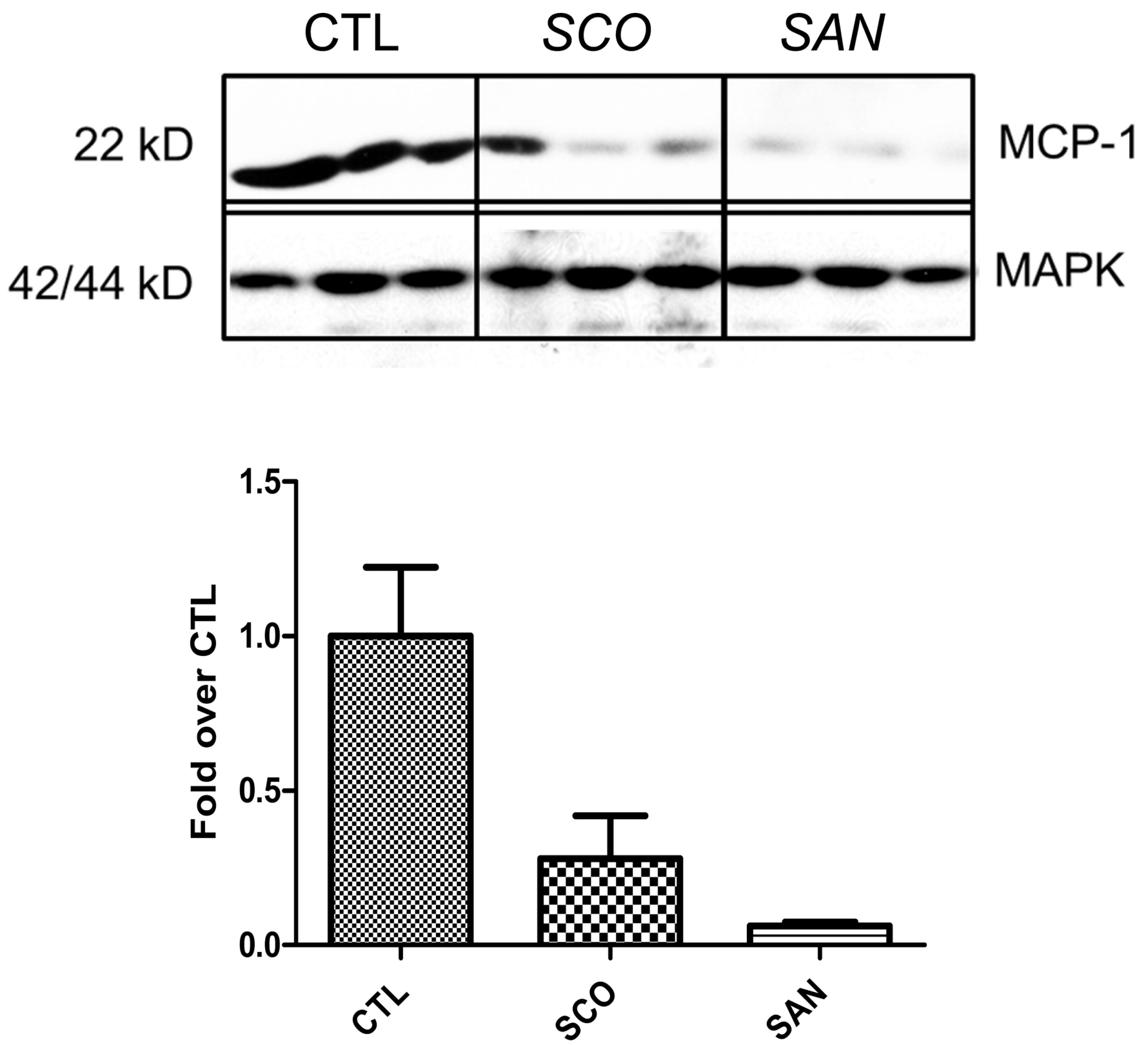

Figure 6. SCO and SAN decrease MCP-1 levels in WAT

rWAT from CTL-, SCO-, and SAN-treated mice was subjected to Western blot analysis (100 $\mu \mathrm{g}$ total protein/lane). Densitometric analysis is shown below the blot; MCP-1 band intensities were normalized to the respective MAPK band intensities prior to comparison against CTL. Data are means $+/-\operatorname{SEM}(\mathrm{n}=3)$. 